\title{
From Cirrhosis to Hepatocellular Carcinoma: New Molecular Insights on Inflammation and Cellular Senescence
}

\author{
Gayatri Ramakrishna ${ }^{a} \quad$ Archana Rastogi $^{b}$ Nirupama Trehanpatia \\ Bijoya Sen ${ }^{a}$ Ritu Khoslaa Shiv K. Sarin ${ }^{c}$ \\ aDepartment of Research, b Department of Pathology and 'Department of Hepatology, \\ Institute of Liver and Biliary Sciences, New Delhi, India
}

\section{Key Words}

Cellular senescence · Cirrhosis - Genetic/Epigenetic landscape ·

Hepatocellular carcinoma $\cdot$ Inflammasomes

\begin{abstract}
Sequential progression from chronic liver disease to fibrosis and to cirrhosis culminates in neoplasia in hepatocellular carcinoma (HCC). The preneoplastic setting of the cirrhotic background provides a conducive environment for cellular transformation. The role of classical inflammation in cirrhosis is widely known, but the exact mechanism linking inflammation and cancer remains elusive. Recent studies have elucidated roles for NF-KB, STAT3 and JNK as possible missing links. In addition, the "inflammasome" (a multiprotein complex and sensor of cellular damage) is a recently identified player in this field. The hallmarks of cirrhosis include necroinflammation, deposition of extracellular matrix and shortening of telomeres, leading to senescence and regeneration. Additionally, the accumulation of genetic/epigenetic changes propels atypical cells toward a malignant phenotype. This review provides recent information on the classical inflammatory pathway, together with a spotlight on inflammasomes and the immunomodulatory role of cellular senescence during the progression from cirrhosis to HCC. Moreover, lacunae in the current knowledge were identified and key questions raised on whether the observed adaptive responses are beneficial or detrimental to tissue homeostasis in a complex organ like liver.

Copyright (C) 2013 S. Karger AG, Basel
\end{abstract}




\section{Introduction}

Hepatocellular carcinoma (HCC) is a complex disorderly state involving multiple events and etiologies, typically viral hepatitis and metabolic syndrome. Intriguingly, the incidence of HCC in men is four times that in women [1]. The majority of cases occur against a background of chronic inflammation, and this has been confirmed by several clinical and epidemiological studies. The occurrence of HCC is often associated with multiple risk factors such as persistent infection with hepatitis virus, chronic alcohol consumption and aflatoxin B1 exposure. Additionally, metabolic disorders such as diabetes and obesity are also considered risk factors for liver cancer. Regardless of the etiology, the neoplastic lesion usually originates on a bed of chronic necroinflammation that sequentially progresses from fibrosis to cirrhosis and finally culminates in HCC (fig. 1, 2). However, a small fraction of cases occur in the absence of cirrhosis. Like any other neoplasia, the development of HCC is a multievent process involving series of genetic alterations, such as mutations and copy number variations, and epigenetic events such CpG island methylation and histone acetylation/ deacetylation that lead to deregulated expression of various genes. Since cirrhosis precedes neoplastic transformation, it is important to understand the key hallmarks of cirrhotic liver, which include necroinflammation and telomere shortening, which lead to senescence and the appearance of regenerating nodules. Each of these apparently adaptive features in liver pathology (which sometimes exhibit opposing functions) plays a role in the progression to neoplasia.

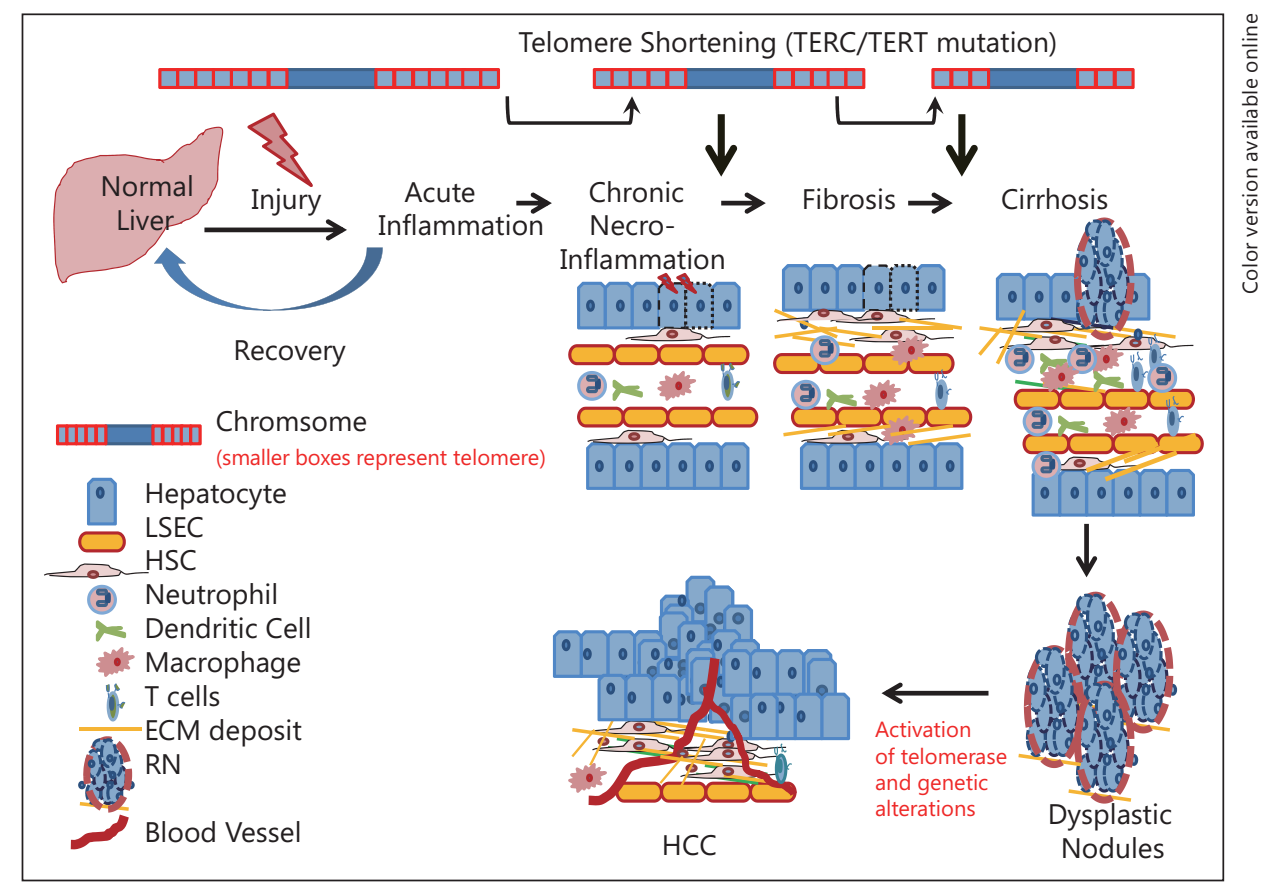

Fig. 1. Diagrammatic representation of various pathological changes associated with liver disease progression. Necroinflammation and telomere shortening are hallmarks of the early stages of chronic liver disease that finally lead to fibrosis and cirrhosis. Some regenerating nodules in the cirrhotic liver show atypical cells that progress toward dysplasia and culminate as a neoplastic lesion. Neoplasia is accompanied with telomerase activation and accumulations of genetic/epigenetic alterations (see fig. 5). 




Fig. 2. Chronological representation of histopathological events during progression toward HCC. The majority of HCC lesions occur against a cirrhotic background (green asterisk), whereas in rare instances it can also occur against a noncirrhotic background (red asterisk). Typical histopathology sections during disease progression are shown: (a) normal liver $(\times 200)$, (b) chronic hepatitis with marked portal/periportal chronic inflammation $(\times 100)$, (c) cirrhosis with regenerating parenchymal nodules separated by active fibrous septa $(\times 40)$, (d1) ductular reaction in cirrhosis $(\times 100)$, (d2) ductular reaction with different stages of differentiation toward hepatocytes in a regenerating nodule $(\times 200)$, (d3) large cell changes in regenerating nodule in cirrhosis $(\times 100)$, (e1) large cell dysplasia $(\times 200)$, (e2) small cell dysplasia $(\times 200)$, (f1) HCC with thick trabeculae of malignant hepatocytes $(\times 200)$ and (f2) HCC with trabeculae and pseudoacinar transformation in a noncirrhotic background $(\times 40)$.

\section{Inflammatory Bed, Inflammasomes and Regenerating Nodules during Cirrhosis as a Prelude to HCC}

A link between chronic inflammation and cancer was first proposed by Rudolf Virchow in 1863. This link was revisited in the recent seminal theory on hallmarks of cancer as proposed by Hanahan and Weinberg, wherein inflammation is now considered an important factor leading toward cancer progression [2]. Local inflammation in hepatic tissue is driven by infiltrating immune cells (monocytes/macrophages, T lymphocytes and neutrophils) and also by resident liver nonparenchymal cells [Kupffer cells, dendritic cells, liver sinusoidal cells and hepatic stellate cells (HSCs)]. In a complex organ such as the liver, different cell types can secrete diverse cytokines/chemokines, and the resulting cocktail constitutes a "secretome" that leads to immunomodulation that manifests as an acute or chronic inflammatory response. Chronic inflammation acts as a favorable preneoplastic setting. 


\section{The Classical Inflammatory Response}

Inflammation due to liver injury plays an important etiological role in hepatobiliary cancer regardless of the presence or absence of microbes (bacteria and hepatitis viruses) or endotoxins. Two classes of inflammation occur in the context of liver: persistent inflammation as a result of hepatitis virus or microbial attack resulting from breaches of the gut-liver axis; sterile inflammation associated with alcohol abuse, nonalcoholic steatohepatitis (NASH) and drug-induced liver injury.

The acute inflammatory response occurs immediately or in minutes, hours or days following injury. Normally, this is a physiologically beneficial response that helps in clearing injured hepatocytes and leads to wound healing. When this process fails, an overdrive of immune cells occurs that perpetuates as chronic inflammation [3]. As the name suggests, chronic inflammation is a prolonged progressive process lasting for months that tilts the homeostasis more toward damage than toward healing. In liver, chronic inflammation eventually sets the stage for progression toward cirrhosis and eventually to HCC.

Hepatocyte injury resulting from microbial or sterile etiologies first activates the resident liver immune cells and later facilitates the recruitment of nonresident immune cells to liver, thereby mounting a strong inflammatory response. The strategic location and diverse functions carried out by liver mean that it also acts as a specialized lymphoid organ [4]. The circulatory network of the portal vein, hepatic artery, sinusoids and the gut-liver axis imparts a unique immune environment to liver. Immunologically active resident liver cells include Kupffer cells, liver dendritic cells, T cells, natural killer T cells and natural killer (NK) cells. Additionally, parenchymal hepatocytes and nonparenchymal liver cells such as HSCs and liver sinusoidal endothelial cells (LSECs) can work as antigen presenting cells [5]. Thus, the active immune compartment in liver makes it a major organ associated with strong inflammatory reactions. In general, whenever there is microbial infection, pattern recognition receptors, which include the membrane-bound Toll-like receptors (TLRs) and C-type lectins, are activated to switch on the signaling cascade that drives production of proinflammatory cytokines.

Despite this wealth of background information available on the classical inflammatory response in liver, lacunae exist in our understanding of the molecular link between inflammation and HCC. The transgenic mouse model of inflammation has proven an extremely useful tool in understanding this link. Studies have confirmed the importance of the NF$\kappa B-S T A T 3$ inflammatory nexus as a missing link [6-8]. One important study used mdr2-/mice, which develop cholangitis, chronic liver inflammation and finally HCC [6]. In this inflammatory mouse model, the tumor necrosis factor (TNF)-NF- $\kappa B$ axis was found to have a procarcinogenic effect on liver. Inhibiting the NF- $\mathrm{BB}$ circuitry by either treatment with anti-TNF- $\alpha$ or with induction of its repressor IkB prevented inflammation, thereby inhibiting HCC tumor progression. In another transgenic mouse model, the expression of lymphotoxin (LT) heterodimers caused chronic inflammation with infiltration of T, B and dendritic cells in liver accompanied with elevated production of cytokines [interleukin $1 \beta$ (IL-1 $\beta$ ), interferon $\gamma$ (IFN- $\gamma$ ) and IL-6], eventually culminating in HCC [7]. When these LT transgenic mice were crossed with Ikk $\beta^{\Delta \text { hep }}$ mice, the resulting transgenic mice showed reduced liver inflammation and no occurrence of HCC. Additionally, in LT transgenic mice models, it has been shown that NF- $\kappa$ B activation leads to production of chemokines such as CXCL10 (which attracts T and NK cells), CXCL1 (which attracts T cells, B cells and neutrophils) and CCL7 (which attracts monocytes). This process eventually leads to chemoattraction and immunomodulation favoring hepatitis. Thus, the inflammatory mdr2-/- and LT transgenic mouse models clearly point toward NF- $\mathrm{BB}$ as the link between inflammation and cancer. These findings were corroborated by studies of human HCC in which activation of NF- $\kappa B$ is a frequent and early event, regardless of the etiology $[8,9]$. 


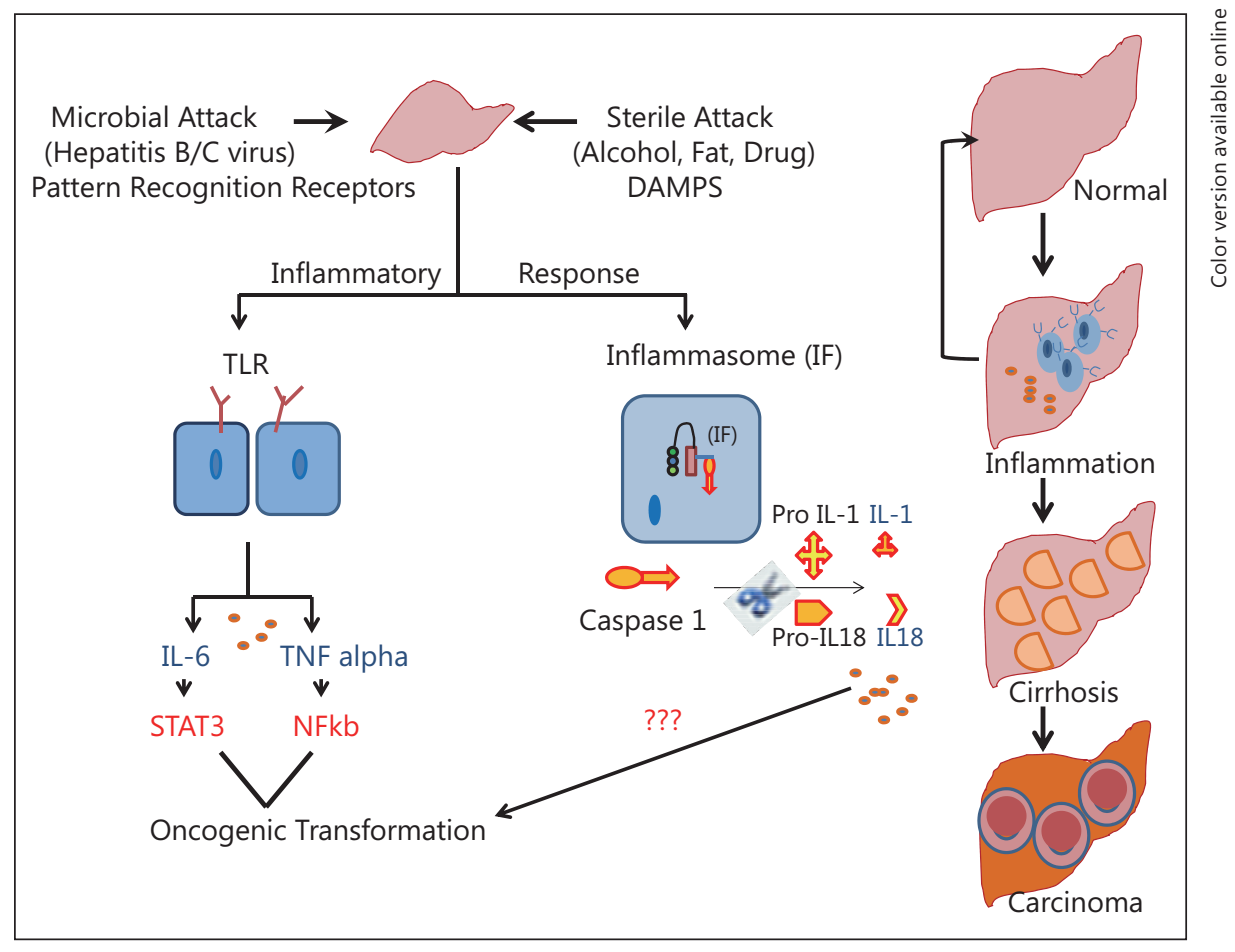

Fig. 3. Inflammation is a hallmark of injured liver. The inflammatory response, caused by viral (microbial attack) or nonviral etiologies (sterile attacks), leads to production of proinflammatory cytokines such as IL6, TNF- $\alpha$, IL1 and IL18 through inflammasome-independent or -dependent pathways. The inflammasome component provides a platform for activation of caspase, which in turn cleaves Pro-IL1/IL18 to their active forms. Proinflammatory cytokines, through activation of transcription factors or by yet unknown mechanisms, make the hepatic milieu a fertile zone for cellular transformation. The accompanying pathological stages are shown in the right panel. DAMPS= damage-associated molecular patterns.

In a fairly recent development, transcription target STAT3 was reported as another crucial link between inflammation and HCC progression [10]. Using mouse models of noninfectious processes such as obesity, it has been shown that proinflammatory cytokines such as IL6 and TNF lead to activation of downstream target STAT3, which then pushes the liver microenvironment toward an oncogenic setting, thereby promoting neoplastic transformation (fig. 3) [10]. Recent findings show that male mice lacking IL6 (-/-) and TNFR (-/-) are protected from obesity-promoted liver tumorigenesis. Interestingly, STAT3 is preferentially activated in human HCC, but not in the surrounding normal hepatocytes, and STAT3 activation is correlated with tumor aggressiveness [11].

Work led by Roger Davies and colleagues has elegantly dissected the complex dual role of c-Jun N-terminal kinase (JNK) in liver inflammation and carcinogenesis. Briefly, their work showed that JNK activation in the nonparenchymal compartment leads to inflammation, while in the parenchymal compartment JNK activation prevents the development of HCC [12, 13]. Their work used a mouse model to reveal that JNK activation in hematopoietic cells, but not in hepatocytes, plays an active role in production of inflammatory cytokine TNF- $\alpha$ in hepatic tissue [12], thereby leading to hepatitis. More recently, the same team indicated that the pro-inflammatory role of JNK results from macrophage polarization toward the M1 subtype, which induces an inflammatory Th1 immune response [14]. In contrast, they also reported that JNK activation in the parenchymal compartment promotes hepatocyte survival and, by preventing regenerative events, promotes tumor suppression [13]. 
The gender disparity in the incidence of HCC has also proven a fascinating link between proinflammatory cytokine IL6 and the higher cancer incidence in males [15, 16]. Using the carcinogen diethylnitrosamine (DEN)-treated rodent model, it has been shown that male mice with HCC exhibited higher levels of IL6 in serum compared to female mice, which are resistant to DEN-induced tumors [15]. This report concluded that "IL-6 ablation abolishes the male bias in DEN-induced HCC development, while ovariectomy enhances IL-6 production and augments HCC induction in female mice." The fact that IL6 is mainly produced by Kupffer cells raises a question regarding gender bias in Kupffer cell activation that has not currently been addressed. The liver is a sexually dimorphic organ, and gene expression patterns are distinct in male and female liver $[17,18]$. Is there, one wonders, a gender bias in the immune response itself? It was recently hypothesized that X-linked microRNAs may provide an immunological advantage to females [19]. Dissection of the gender bias in the proinflammatory response with respect to preferential microRNA or transcription factor activation and its role in the progression from cirrhosis to HCC remains a challenge to be addressed.

In recent years, the role of infiltrating monocyte-derived macrophages in inflammation and fibrosis has received much attention, in addition to the already well-studied aspects of the resident innate Kupffer cells, dendritic cells and NK and NKT cells [5]. Human monocytes are classified as classical (CD16+CD16-), intermediate (CD14+CD16+) and nonclassical (CD14dimCD16+) types [20]. The different scenarios of acute and chronic inflammation may differ depending on the trafficking of monocytes and the subset ratio of monocytes at the site of inflammation [21]. Nonclassical monocytes are recruited and drive liver fibrogenesis during chronic inflammation of liver [22]. Further, higher expression levels of CCR2, CCR1 and CCR5 have been observed in fibrotic and cirrhotic livers. It has been also noted that perpetuation of chronic inflammation occurs due to a preferential differentiation of monocytes into nitric oxide synthase-producing macrophages, which in turn exert profibrogenic actions by activating HSCs [23]. It remains to be seen whether the peripheral monocyte subset can be used as a diagnostic/screening marker for liver disease progression and whether therapeutic modulation of chemokines will affect the monocyte subset ratio to prevent chronic inflammation. A role for LSECs in liver inflammation has been proposed. In normal liver, LSECs actively prevent fibrosis by maintaining HSCs in a quiescent state [24]; however, in hepatic fibrosis, they promote inflammation by inducing a strong cytotoxic $\mathrm{T}$ cell response. LSECs themselves do not produce inflammatory cytokines; however, they induce T cells to produce proinflammatory mediators such as IL-6, IFN- $\gamma$, TNF- $\alpha$ and MCP-1 [25].

Accumulating data suggest the involvement of naturally occurring $\mathrm{CD} 4^{+} \mathrm{CD} 25^{\text {high }}$ regulatory $\mathrm{T}$ cells (Tregs) in cirrhosis. Reports of chronic inflammation in autoimmune disorders such as rheumatoid arthritis suggest that increased infiltration of Foxp3+Tregs in damaged organs and at the sites of inflammation has a suppressive role in combating injurious inflammation [26]. In nonautoimmune diseases such as chronic viral hepatitis and primary biliary cirrhosis, studies have suggested that Tregs are involved in the maintenance of chronic inflammation $[27,28]$. In support of the latter finding, recent work from our group has indicated a role for Tregs coupled to activation of Notch and TGF- $\beta$ in perpetuation of the inflammatory response during liver disease progression in cirrhosis and HCC in chronically hepatitis B virus (HBV)-infected patients [27] (fig. 4).

Based on the above background information, it is evident that inflammation, through activation of classical pathways, is a distinct feature of most states of liver disease, regardless of its etiology. Acute inflammation is protective, whereas chronic inflammation initiates necroinflammation, tissue remodeling and an oxidative microenvironment. Oxidative bursts trigger DNA damage and genomic aberrations that finally culminate in neoplasia. The challenge remains of identifying the nature of immune/nonimmune cells and the cocktail of chemokines/cytokines constituting the secretome that can differentiate acute versus chronic 


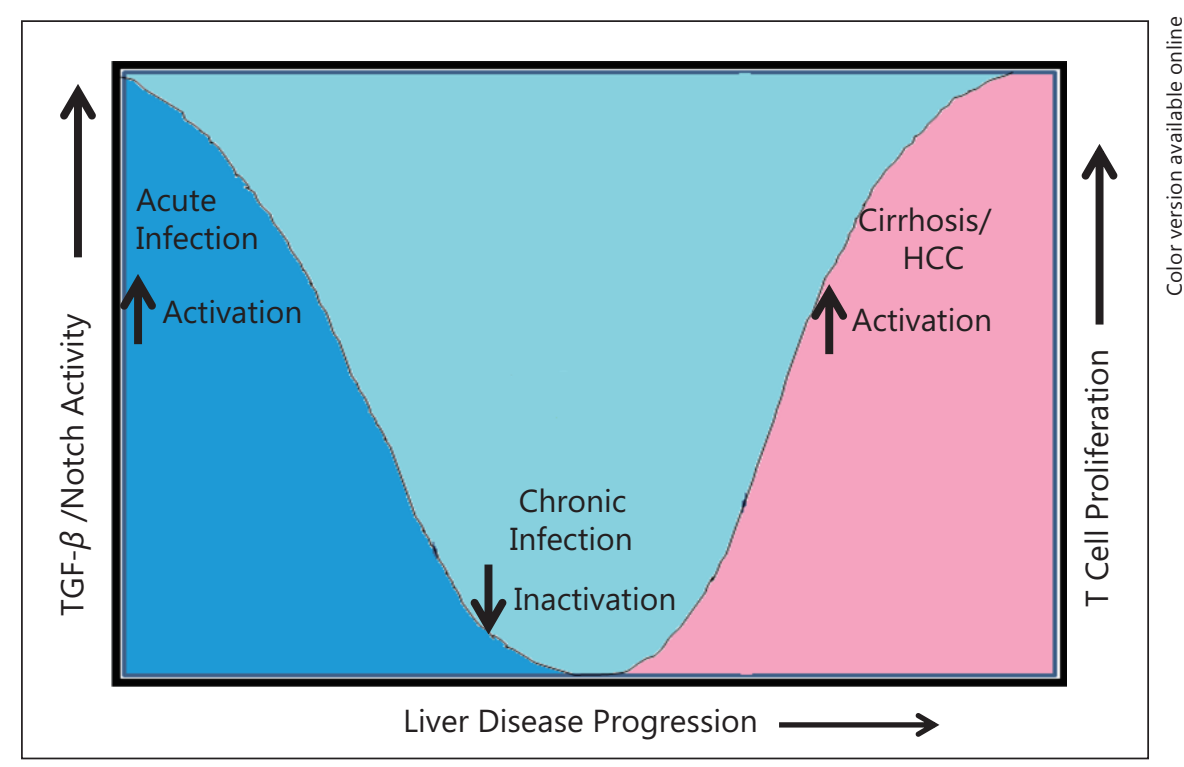

Fig. 4. Graphical representation showing Notch/TGF- $\beta$ signaling and T cell activation during liver disease progression through phases of acute and chronic HBV infection with respect to the later stages of end-stage liver disease, i.e., cirrhosis and HCC. Activation is seen during the acute and end stages of liver disease, whereas attenuation is noted during the chronic stage of infection [28].

inflammatory responses in different settings of liver disease, e.g., fibrosis, cirrhosis, dysplastic nodules and neoplasia. Future goals should include therapeutic strategies to prevent chronic inflammation and to divert tissue homeostasis toward normalcy. A pertinent step would be to exploit NF- $\mathrm{kB}$, JNK, STAT3 and monocyte polarization as therapeutic targets to prevent chronic inflammation and prevent disease progression.

\section{Inflammasomes}

Recent studies found that the immune compartment includes a specialized intracellular multiprotein complex called the inflammasome, which fosters the maturation of proinflammatory cytokines such as IL-1b and IL18 needed for the innate immune response and tissue homeostasis [29]. Inflammasomes essentially function as sensors, monitoring the signals instigated by tissue injury or dying cells, thereby recognizing the intracellular danger signals via NOD-like receptors (NLRs) [30]. NLRs have three defined domains: the leucine-rich-repeat (LRR) domain that binds the ligands, an oligomerization domain called NACHT with dNTPase activity, and a caspase recruitment domain (CARD). The LRR domain senses ligands that include pathogen-associated molecular patterns and molecules released during tissue injury, such as extracellular ATP, hyaluronan, saturated fatty acids amyloid- $\beta$ fibrils and uric acid [31]. Once a danger signal is sensed by NLRs, it oligomerizes via the NACHT domains, thereby recruiting pro-caspase-1, which in turn activates caspase- 1 . Thus, inflammasomes act as specialized platforms for active caspase- 1 to cleave its substrates IL-1 $\beta$ and IL-18 (fig. 3). Inflammasome activation, in turn, is responsible for local and systemic inflammatory reactions, recruitment of neutrophils and platelets and activation of the innate immune system [32,33].

Inflammasomes are not restricted to immune cells but constitute an important sensing mechanism in the nonimmune compartment also. In the context of hepatic tissue, multimeric inflammasome components are present in both parenchymal and nonparenchymal cells [34]. Briefly, the different inflammasomes are categorized as NLRP1, NLRP3, NLRC4 and AIM2. Of these different types, NLRP3 occurs prominently in hepatocytes [35], stellate cells [36] and 




Liver Cancer 2013;2:367-383

DOI: $10.1159 / 000343852$

Published onlıne: August 26, 2013

sinusoidal endothelial cells [37]. Kupffer cells are a major source of IL-1 [38], thereby indicating the importance of inflammasomes in initiating the pro-inflammatory response. High expression of inflammasome components in fibrotic lesions was found using animal models involving $\mathrm{CCl}_{4}$ treatment or bile duct ligation [33].

The role of the inflammasome has been now demonstrated in acetaminophen- [37] and LPS-induced liver injury [39], viral hepatitis [40] and more elaborately in alcoholic and nonalcoholic fatty liver disease $[35,41,42]$. The proof of concept for involvement of inflammasomes in liver disease progression comes from studies on knockout mice in which different components of the inflammasome were deleted. Some contradictions exist in the findings of these model studies. Studies using the acetaminophen liver injury model show liver protection in NLRP3 inflammasome (ASC, NLRP3, caspase-1) knockout mice [37]. In contrast, the absence of inflammasome components $\left(\mathrm{Asc}^{-/-}\right.$, and caspase-1-/- and NLRP3-/- mice) enhances the progression of nonalcoholic fatty liver disease and NASH in mice fed with a methionine-choline-deficient diet [42]. Additionally, it was shown that the absence of inflammasomes affects the gut-liver axis as it resulted in microbial dysbiosis and inflammation in liver through the influx of Toll-like receptor (TLR) 4 and TLR9 agonists into the portal circulation [42].

These contrasting findings on inflammasomes and liver disease progression can be explained by the complexity of the pro-inflammatory multimeric components, the cell type in which it gets activated and the different etiologies of liver injury. Nonetheless, it is clear that inflammasome-dependent processing of the proinflammatory response is intricately associated with a pro/anti response in the context of liver damage either dependent or independent of the gut-axis connection. Future studies with knockout mice will throw more light on inflammasome-mediated protective versus promotive effects in liver neoplastic progression.

\section{Ductular Reaction, Regenerating Nodules and the Genomic and Epigenomic Landscape for HCC Development \\ HCC almost always occurs in a histologically abnormal liver. Overtly active immune cells} in chronic settings produce a proinflammatory cytokine storm that results in tissue injury as a result of hepatocyte cell death. Commonly referred to as necroinflammation, this phenomenon is characterized by increased ductules around the portal tract with accompanying inflammatory cells and stromal changes [43]. This ductular reaction has gained prominence because it indicates a regenerative response by intrinsic liver cells. In fact, the proximal branches of the biliary tree, including the bile ductules and the canals of Hering, constitute a special niche for the stem/progenitor cell compartment [44].

The immediate response to necroinflammation and cellular death is deposition of extracellular matrix (ECM) by activation of HSCs and activation of mature hepatocytes to proliferate and regenerate. However, in severe liver damage, proliferating hepatocytes are unable to regenerate the liver because of cellular senescence or growth arrest [45]. In such scenarios, ductal reactions (DRs) are evoked as a second-line defense by which the reserve progenitor cell population is activated to recoup the damaged hepatocytes. The final manifestation of histologically diverse DRs and parenchymal regeneration occurs in the form of regenerating nodules (RNs) or hepatocyte buds, which are hallmarks of cirrhotic liver [44, 46]. The molecular mechanisms, the cytokine/chemokine cocktail of the inflammatory secretome and the subset of immune cells that promote initiation of DRs and RNs remain to be elucidated.

An important question in the context of RNs is the origin of the cells. The cells within RNs express markers of hepatic (HepPar1) and biliary lineages (cytokeratin 19), depending upon the degree of proliferation and differentiation of hepatic progenitor cells [47]. The regenerating nodules can be either polyclonal or monoclonal in origin [48]. The monoclonal 
origin of RN cells was reported by Lin et al [49], who stated that the cells of origin are more likely to be a facultative stem cell from the biliary tree.

It is now well accepted that cirrhosis is often a prelude to HCC, irrespective of the underlying etiology, and this leads to the question whether RNs are premalignant lesions. In general the $\mathrm{RN}$ is benign in nature. However, with the passage of time, they are increasingly likely to develop into dysplastic nodules, which represent a premalignant state [50]. Intriguingly, not all dysplastic nodules progress to neoplasia, and it was recently reported in a case-control study that low- or high-grade dysplastic nodules either regressed or remained static [51].

Then what gives the growth advantage to hepatocytes in the RN that leads toward neoplasia? The exact molecular mechanism initiating the RN is still unclear, but generally it is believed that deposition of ECM triggers activation of progenitor cells. The role of various hepatocyte mitogens (e.g., EGF, HGF, TGF- $\alpha$ and amphiregulin) in hepatocyte proliferation is known, yet there is no one single mechanism attributed to HCC development because of the complexity of the disease. The rapid rate of cellular proliferation in a milieu of inflammatory background predisposes the hepatocytes or liver stem cells to accumulate DNA damage and, when left uncorrected, this leads to accumulation of genetic/epigenetic alterations [52, 53]. The genomic aberrations in RNs will thereby transform the preneoplastic lesion to dysplastic nodules, and finally the malignant transformation to early HCC will take place.

With the advent of new technologies in the postgenomic era, rapid progress has been made in identifying genomic aberrations that lead to progression toward neoplastic growth (fig. 5). In the context of liver, polymorphisms in genes such as IFN- $\gamma, T N F-\alpha, I L-10, M C P 2$ and factor V Leiden have been associated with progression from fibrosis to cirrhosis [54-56]. Using a genomic scan approach, a seven gene variant signature was found to be associated with progression toward cirrhosis in HCV-infected patients [57]. The cirrhosis risk score (CRS) is based on the occurrence of single nucleotide polymorphisms (SNPs). The seven signature SNPs include following genomic locations: Toll-like receptor 4 (TLR 4), syntaxin binding protein 5-like (STXBP5L), antizyme inhibitor $(A Z I N)$, transient receptor TRPM5, potential cation channel $A Q P 2$ subfamily member, the intergenic region between degenerative spermatocyte homolog 1(DEGS1) and nuclear valosin-containing protein-like $(N V L)$. The seven-gene signature was found to be a useful prognostic marker for progression of HCV-associated liver disease [58]. It is not yet clear whether predictions based on CRS using the same SNP signature will hold true for other chronic liver disease with etiologies such as HBV or steatosis.

Previously, p53 mutations were noted in RNs of cirrhotic liver with an incidence of 7.7$58.8 \%$, depending on the nodule. More recently, two studies have shown mutations in telomerase genes TERT and TERC in cirrhosis, albeit at a lower frequency [59-61]. Importantly, epigenetic silencing resulting from CpG methylation, particularly in the tumor suppressor genes, has also been noted in liver disease. In this regard, the most frequently altered gene is $R A S S F 1 A$, for which promoter methylation is noted at high frequency during progression from cirrhosis to dysplastic nodules to HCC $[62,63]$. An increased frequency in CpG island methylation has also been noted for genes such as $p 16$ and COX2 in HBV-infected dysplastic nodules and HCC [63].

The following key points have emerged from recent landmark studies that evaluated the genetic landscape of the HCC exome using next-generation sequencing: (a) the most frequent mutations ( $48 \%$ of HCC cases) occurred in the Wnt/ $\beta$-catenin pathway with either activating mutations in $\beta$-catenin (32\%) or inactive mutations in AXIN1 or APC $(16 \%)$; (b) alterations in p53/cell cycle check point control were the next most frequent in HCC, with inactivations in the TP53 gene and encoding interferon regulatory factor 2 (IRF2) which interacts with $\mathrm{mdm} 2$, an inhibitor of p53; (c) the third most frequent alterations occurred in the chromatin remodelers ARID1A and ARID2; and (d) the least altered genes appeared in the PI3 K/Ras signaling pathway and those involved in oxidative stress $[64,65]$. In support of these findings, work 


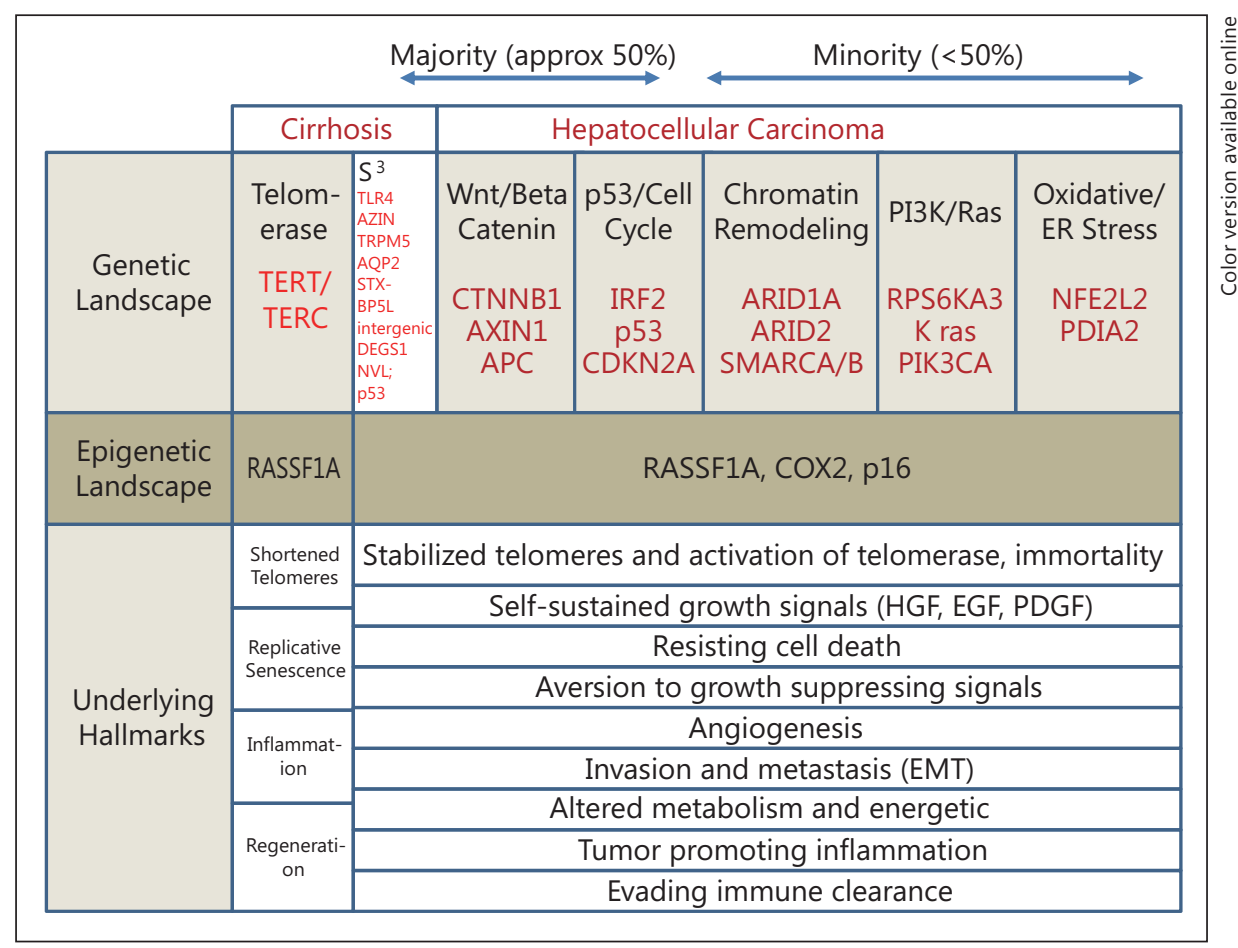

Fig. 5. Genetic/epigenetic landscape together with associated hallmarks of cirrhotic liver and HCC. $\mathrm{S}^{3}$ refers to the seven signature single nucleotide polymorphisms that are usually seen in HCV-related cirrhosis, in addition to mutation in P53. EMT = epithelial-to-mesenchymal transition. The classification of genetic changes in HCC is as has been described by Guichard et al [64].

published from our own group has suggested infrequent RAS mutations in HCC; however, we found unusually low levels of Ras protein in HCC, suggesting the involvement of wild-type $R A S$ as a possible tumor suppressor [66].

To summarize the first section of this article, it is apparent that parenchymal/nonparenchymal compartments, either through direct TLR activation or through inflammasomes, promote the generation of local inflammatory bursts in response to tissue injury for wound healing and resolving. However, a persistent inflammatory response caused by overtly active immune cells results in oxidative DNA damage leading to genomic/epigenomic alterations, thereby affecting cellular metabolism and making conditions favorable for progression toward neoplasia. The challenging issues ahead involve identification of dysregulated immune cell subset together with their gene expression signatures and the inflammatory secretome involved in the stepwise progression from inflammation to cirrhosis leading to neoplasia in liver.

\section{Cellular Senescence: The Yin and Yang in HCC Progression}

Cellular senescence reflects a condition of permanent growth arrest from which the cell cannot reenter the cell cycle $[67,68]$. There are two basic mechanisms for inducing senescence. First, telomere attrition with each round of cell division makes a cell lose its proliferative potential, thereby exiting the cell cycle; this is commonly referred to as replicative senescence. Replicative senescence is usually a slow process, and in in vitro primary cell culture conditions it is also referred to as the Hayflick limit. Second, oxidative damage or 
oncogenes induce permanent growth arrest in an accelerated fashion, and this is commonly referred to as stress/oncogene-induced premature senescence [69]. In liver, both replicative and oncogene-induced senescence has been noted in both the parenchymal (hepatocytes) and nonparenchymal compartments (HSCs) [70-72]. Recent developments have identified a link between cellular senescence and cancer [73]; in fact, senescence seems to act as a doubleedged sword. Senescence of nonparenchymal cells such as HSCs plays a part in maintaining tissue homeostasis by helping resolve fibrosis. The accumulation of senescent parenchymal cells is a necessary event in progression toward cirrhosis and HCC; in contrast, however, senescence in transformed hepatocytes causes growth arrest and hence is considered an antitumorigenic mechanism [70, 73, 74] ( fig.6). Therefore, it is a conundrum in liver biology as to whether senescence is detrimental or beneficial to liver. We review below human and rodent studies elucidating the role of senescence in liver depending on the pathology.

Beneficial Effects of Cellular Senescence by Immunomodulation: Resolution of Fibrosis and Senescence Surveillance for Limiting Neoplasia

The process of cellular senescence in HSCs is intimately involved in liver tissue homeostasis. HSCs reside in the space of Disse and are usually quiescent cells that become activated during liver injury. Activated HSCs are involved in the fibrotic response during wound healing. This response involves the following steps: (a) activation and transdifferentiation of HSCs to myofibroblasts that are $\alpha$-SMA positive, (b) ECM deposition by activated cells, including secretion of collagen and TIMP to fill the wound and (c) finally, when the wound is filled, the activated HSCs are cleared either by apoptosis or by undergoing cellular senescence to be later eliminated by immune cells. The clearing of HSCs forms an important part of resolving fibrosis $[74,75]$.

In general, the process of cellular senescence involves activation of tumor suppressors p53 and p16. Lowe and co-workers, using a $\mathrm{CCl}_{4}$ model of cirrhosis with compromised p53 or p16, reported that in the absence of senescence, overtly activated HSCs continue to deposit ECM, leading to severe hepatic fibrosis [70]. Additionally, the same team showed that senescent HSCs are beneficial because they exert a strong immunomodulatory effect that helps in the recruitment of other immune cells such as macrophages at the site of tissue injury. The recruited immune cells, in turn, clear up the senescent cells and also help in dissolution of the fibrotic lesion. Thus, these senescent cells have an antifibrotic role that is beneficial for wound healing and resolution [69-71].

Another beneficial attribute of senescing hepatocytes is their antitumorigenic role in liver. This is supported by recent work using a mouse model in which adenoviral delivery of oncogenic Ras induced hepatocyte senescence that in turn led to immunomodulation by attracting various immune cells such as macrophages, CD4 T cells, neutrophils and NK cells to the site of hepatocyte damage and clearing the premalignant lesion [72]. A characteristic feature of senescent cells is the secretion of various chemokines/cytokines such as interleukins, RANTES and CXCL10, i.e., the secretome of senescent cell makes them good immunomodulators [72]. The protective nature of the senescence-associated secretory phenotype phenomenon has now been termed "senescence surveillance" because it helps in selective clearance of neoplastic cells in the liver. Thus, senescent cells are now considered antitumorigenic because they restrict the growth of neoplastic cells by permanent cell cycle arrest, and the senescent secretome attracts immune cells to clear the neoplastic cells. Interestingly, under in vitro conditions, tumorigenic HCC cell lines such as Huh7 can undergo premature senescence when treated with DNA damage drugs such as adriamycin/doxorubicin (fig. 7a). Since senescent cells are permanently growth arrested, this property could be exploited in the future to stop the growth of neoplastic cells by treating tumor cells with DNA damage chemicals that can induce premature senescence. 


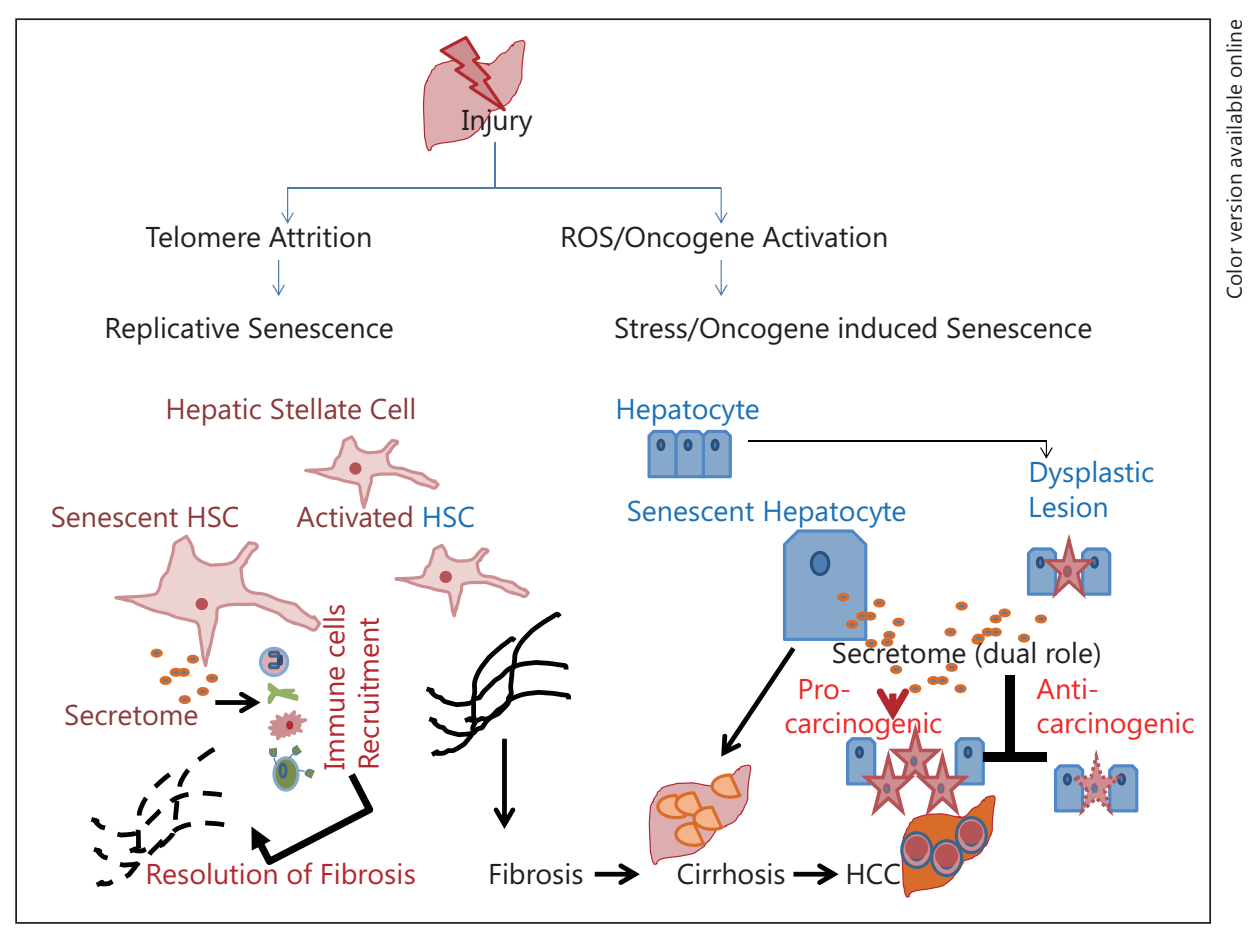

Fig. 6. Opposing roles of cellular senescence depending on cell type and the pathological state of the liver. The secretome phenotype of senescent cells makes them active immunomodulators. In a cirrhotic background, the senescence of HSCs is beneficial for the resolution of fibrosis; in contrast, accumulation of hepatocyte senescence is a hallmark of cirrhosis. The senescent hepatocyte, through its secretome activity, can help in clearing neoplastic cells and thus have an anticarcinogenic effect; however, in later stages of disease progression, the secretome can act as a procarcinogen influencing the proliferation of neighboring parenchymal cells or stem cells. ROS = reactive oxygen species.

Detrimental Effects of Hepatocyte Replicative Senescence in Progression of Chronic Liver Disease to Cirrhosis and Its Possible Procarcinogenic Effects

The occurrence of senescent cells in ageing and the development of cirrhosis points to their role in underlying pathology $[73,77]$. Hepatocyte senescence is intricately linked to telomerase and telomere biology. Telomeres are specialized structures at the ends of chromosomes, and with each round of cell division the telomeres shorten. This shortening of telomeres represents an internal biological clock that determines the process of cellular or organismal ageing.

Cellular ageing, which is generally referred to as replicative senescence, has been noted in the cirrhotic liver. The key points that have emerged are (a) progressive shortening of telomeres occurs during the chronology of events leading to cirrhosis in the following order: chronic hepatitis > fibrosis > cirrhosis, with the shortest telomeres in cirrhotic liver and (b) mutations in the telomerase genes TERT (7\% incidence) and to a lesser extent TERC are associated with cirrhosis $[60,61]$. Following tissue injury leading to loss of parenchymal cells, the first line of defense is activation of quiescent hepatocytes to restore the lost cells. There is considerable shortening of telomeres because adult differentiated hepatocytes are low in telomerase activity. Eventually, the dividing hepatocytes undergo growth arrest due to senescence. Telomere shortening has emerged as a hallmark of cirrhotic liver, regardless of mutations in telomerase genes.

This link of shortening of telomeres with replicative senescence in hepatocytes of cirrhotic liver is further substantiated by the presence of growth arrest senescence markers 

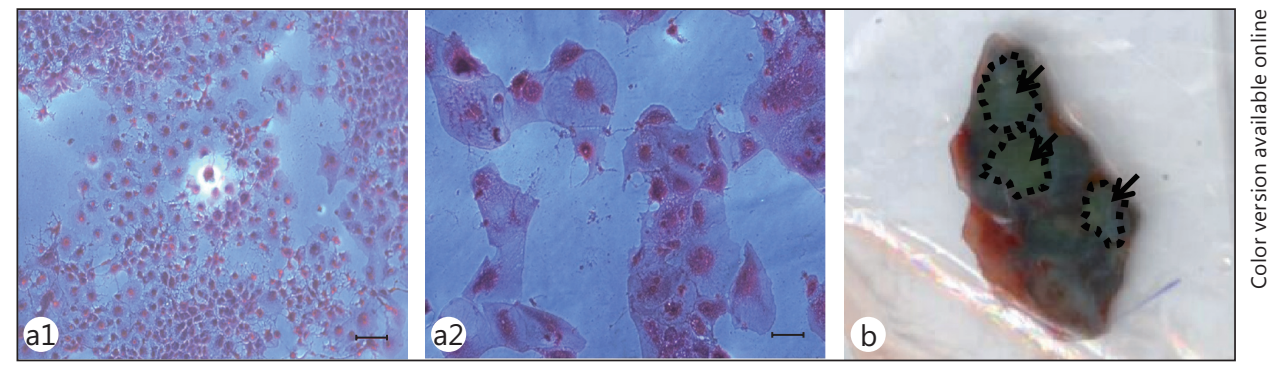

Fig. 7. Hepatic senescence. (a) HCC cell line Huh7 without (a1) or with doxorubicin treatment (a2). Note that the senescent cells (a2) showed an enlarged morphology, a typical feature of stress-induced premature senescence, as a result of the action of doxorubicin. Cells were stained with hematoxylin and eosin. Scale bar $=10 \mu \mathrm{M}$. (b) Cirrhotic liver tissue stained positive for senescent associated $\beta$-galactosidase activity. The dotted lines and arrow indicate the cirrhotic nodules. Note the blue staining in the cirrhotic liver.

such as p21, p27 and SA- $\beta$-galactosidase. This was confirmed in our laboratories on directly subjecting explanted cirrhotic liver tissue to $S A-\beta$-galactosidase staining. The cells in cirrhotic tissue took an intense blue stain indicative of cellular senescence (fig. 7b). Besides cirrhosis, the phenomenon of senescence is also detected in liver tissue with progressive ageing. Rodent liver from older animals ( 2 years and above) showed accumulation of hepatocytes with typical features of senescence such as enlarged nuclei, increased polyploidy and accumulation of oil droplets [76].

Is the accumulation of senescent cells detrimental in settings of ageing and cirrhosis? Because senescent parenchymal cells have compromised liver function, the accumulation of senescent cells may damage normal tissue homeostasis. ?Additionally, the immunomodulatory effects of senescent cells, as well as being beneficial, can also have a procarcinogenic effect, and this is supported by the following facts: (a) the proinflammatory cytokines and chemokines secreted by senescent cells can favor uncontrolled proliferation of a nearby premalignant hepatocyte or stem cell, (b) senescent cells can influence the epithelial-to-mesenchymal transition in parenchymal cells, thereby promoting metastasis and (c) senescent cells can modulate macrophage activity in a way that may promote abnormal blood vessel development leading to angiogenesis and disease progression. It remains to be seen if any of the above-mentioned processes modulated by senescent cells occur in the liver microenvironment either during ageing or cirrhosis, thereby promoting HCC development.

In conclusion, it is apparent that the earlier paradigm that senescent cells are simply permanently retired and resting is no longer true. It is now evident that senescent cells are specialized cells with a strong immunomodulatory phenotype that controls diverse functions depending on the pathological state of the tissue. A senescent parenchymal or nonparenchymal cell in a premalignant or benign setting can have beneficial effects in terms of resolution of fibrosis or the clearing of prospective malignant cells, thereby working as an "anticancer bullet." On the other hand, if parenchymal cells, which constitute the bulk of the liver, undergo senescence en masse, it will lead to compromised liver function and will be deleterious. Consequently, senescence appears to be a double-edged sword with opposing functions in the complex organ that is the liver [77]. 


\section{Future Directions and Key Questions}

The challenges of handling primary HCC, a condition associated with high levels of morbidity and mortality, are: the identification of biomarkers for screening, determining new therapeutic management and prevention strategies. Here, we have outlined some insights on the role of inflammation in the context of inflammasomes and senescent cells in understanding the complex progressive nature of HCC against the backdrop of cirrhosis. Some key areas that need further investigation are:

(a) Identification of the individual cell types and the cocktail of secretory cytokines/chemokines (secretome) involved in pro- and anti-inflammatory responses will not only aid in understanding the beneficial effects of acute inflammation but also help in targeted therapeutics directed toward chronic inflammation.

(b) Do the inflammasome components in various cellular compartments of liver have similar or dissimilar functions?

(c) If there is a correlation between inflammasomes and liver disease progression in viral or nonviral etiologies?

(d) Can the components of inflammasomes be used as markers for either diagnosis or for therapeutic targeting?

(e) What is the role of inflammasomes in RNs?

(f) What are the genetic/epigenetic factors that decide the fate of RNs toward the normal regenerative process vis-a-vis dysplastic transformation?

(g) Can better markers be developed for identifying senescent cells in archived specimens in place of the currently used SA- $\beta$-galactosidase, which requires fresh-frozen samples?

(h) Can the secretory phenotype of senescent cells be exploited to help in liver regeneration? This would require identification of the liver-specific "senescence-associated secretome" of cytokines and chemokines that can promote liver regeneration without leading to neoplastic transformation.

(i) In the neoplastic setting, can a "pro-senescent therapy" be exploited to permanently arrest the proliferation of tumorigenic cells and restrict the growth of HCC? Will future chemotherapeutic regimes adopt a provocative paradigm shift in tumor treatment from killing the tumors to making them static?

\section{Conclusions}

The majority of HCCs occur on a background of cirrhosis, which is characterized by a strong inflammatory response and the presence of senescent cells. The beneficial effects of pro-inflammatory cytokines in acute settings turn harmful when immune cells remain overtly active for long durations, as is seen in the chronic setting of cirrhosis. Immune modulation in the liver can also be triggered by nonimmune cells such as senescent HSCs and senescent hepatocytes. The secretome of immune cells and senescent cells is a decisive factor in the prevention or progression of liver disease. The duality of functions in either protecting from or promoting neoplasia has emerged as a key feature of inflammatory processes and cellular senescence. Thus, we need to view each of these biological processes in a holistic way, both from a reductionist and integrative perspective, because the outcomes of these processes can be either deleterious or beneficial depending on the stage of biological development.

Albert Einstein famously stated that "Explanations should be as simple as possible, but no simpler." In biological organization, a process may appear simple at the cellular level, and yet the seemingly opposing effects of the same phenomenon make processes utterly complex at a tissue and an organismal level. Consequently, the dual roles of inflammation and 
senescence as outlined above have to be considered carefully in the context of their future potential use as therapeutic targets for the treatment of liver disease.

\section{Acknowledgments}

GR wrote the manuscript. AR provided the histopathology slides and gave inputs on cellular pathology. NT provided inputs on liver immunology. RK helped with tissue processing. GR and BS performed the senescence assay. SKS provided overall inputs and edited the manuscript. Funding in the laboratory of SKS and NT is supported by ICMR and DBT. Funding in the laboratory of GR is supported by DBT. We acknowledge the help received from Virender, Arun, Dileep and Surinder. We acknowledge the help received from Prof. Ramasarma in critical reading and editing of the manuscript. We thank Dr. Shvetank for helpful discussions on the concept of the inflammasome.

\section{References}

1 El-Serag HB: Hepatocellular carcinoma. N Engl J Med 2011;365:1118-1127.

2 Hanahan D, Weinberg RA: Hallmarks of cancer: the next generation. Cell 2011;144:646-674.

3 Mueller K: Inflammation. Inflammation's yin-yang. Introduction. Science 2013;339:155.

4 Crispe IN: The liver as a lymphoid organ. Annu Rev Immunol 2009;27:147-163.

5 Liaskou E, Wilson DV, Oo YH: Innate immune cells in liver inflammation. Mediators Inflamm 2012; 2012:949157.

-6 Pikarsky E, Porat RM, Stein I, Abramovitch R, Amit S, Kasem S, et al: NF-kappaB functions as a tumour promoter in inflammation-associated cancer. Nature 2004;431:461-466.

7 Haybaeck J, Zeller N, Wolf MJ, Weber A, Wagner U, Kurrer MO, et al: A lymphotoxin-driven pathway to hepatocellular carcinoma. Cancer Cell 2009;16:295-308.

-8 Liu P, Kimmoun E, Legrand A, Sauvanet A, Degott C, Lardeux B, et al: Activation of NF-kappa B, AP-1 and STAT transcription factors is a frequent and early event in human hepatocellular carcinomas. J Hepatol 2002;37:63-71.

$>9$ Luedde T, Schwabe RF: NF-кB in the liver - linking injury, fibrosis and hepatocellular carcinoma. Nat Rev Gastroenterol Hepatol 2011;8:108-118.

-10 He G, Yu GY, Temkin V, Ogata H, Kuntzen C, Sakurai T, et al: Hepatocyte IKKbeta/NF-kappaB inhibits tumor promotion and progression by preventing oxidative stress-driven STAT3 activation. Cancer Cell 2010;17:286-297.

11 He G, Karin M: NF-кB and STAT3 - key players in liver inflammation and cancer. Cell Res 2011;21:159-168.

12 Das M, Sabio G, Jiang F, Rincón M, Flavell RA, Davis RJ: Induction of hepatitis by JNK-mediated expression of TNF-alpha. Cell 2009;136:249-260.

13 Das M, Garlick DS, Greiner DL, Davis RJ: The role of JNK in the development of hepatocellular carcinoma. Genes Dev 2011;25:634-645.

14 Han MS, Jung DY, Morel C, Lakhani SA, Kim JK, Flavell RA, et al: JNK expression by macrophages promotes obesity-induced insulin resistance and inflammation. Science 2013;339:218-222.

15 Naugler WE, Sakurai T, Kim S, Maeda S, Kim K, Elsharkawy AM, et al: Gender disparity in liver cancer due to sex differences in MyD88-dependent IL-6. Science 2007;317:121-124.

16 Prieto J: Inflammation, HCC and sex: IL-6 in the centre of the triangle. J Hepatol 2008;48:380-381.

17 Yang X, Schadt EE, Wang S, Wang H, Arnold AP, Ingram-Drake L, et al: Tissue-specific expression and regulation of sexually dimorphic genes in mice. Genome Res 2006;16:995-1004.

18 Waxman DJ, Celenza JL: Sexual dimorphism of hepatic gene expression: novel biological role of KRAB zinc finger repressors revealed. Genes Dev 2003;17:2607-2613.

19 Pinheiro I, Dejager L, Libert C: X-chromosome-located microRNAs in immunity: might they explain male/ female differences? The X chromosome-genomic context may affect X-located miRNAs and downstream signaling, thereby contributing to the enhanced immune response of females. Bioessays 2011;33:791-802.

20 Ingersoll MA, Platt AM, Potteaux S, Randolph GJ: Monocyte trafficking in acute and chronic inflammation. Trends Immunol 2011;32:470-477.

-21 Tacke F: Functional role of intrahepatic monocyte subsets for the progression of liver inflammation and liver fibrosis in vivo. Fibrogenesis Tissue Repair 2012;5(Suppl 1):S27.

-22 Zimmermann HW, Seidler S, Nattermann J, Gassler N, Hellerbrand C, Zernecke A, et al: Functional contribution of elevated circulating and hepatic non-classical CD14CD16 monocytes to inflammation and human liver fibrosis. PLoS ONE 2010;5:e11049.

23 Karlmark KR, Weiskirchen R, Zimmermann HW, Gassler N, Ginhoux F, Weber C, et al: Hepatic recruitment of the inflammatory Gr1+ monocyte subset upon liver injury promotes hepatic fibrosis. Hepatology 2009;50:261-274. 
24 Deleve LD, Wang X, Guo Y: Sinusoidal endothelial cells prevent rat stellate cell activation and promote reversion to quiescence. Hepatology 2008;48:920-930.

25 Connolly MK, Bedrosian AS, Malhotra A, Henning JR, Ibrahim J, Vera V, et al: In hepatic fibrosis, liver sinusoidal endothelial cells acquire enhanced immunogenicity. J Immunol 2010;185:2200-2208.

-26 Brusko TM, Putnam AL, Bluestone JA: Human regulatory T cells: role in autoimmune disease and therapeutic opportunities. Immunol Rev 2008;223:371-390.

-27 Sasaki M, Ikeda H, Sawada S, Sato Y, Nakanuma Y: Naturally-occurring regulatory T cells are increased in inflamed portal tracts with cholangiopathy in primary biliary cirrhosis. J Clin Pathol 2007;60:1102-1107.

-28 Trehanpati N, Shrivastav S, Shivakumar B, Khosla R, Bhardwaj S, Chaturvedi J, et al: Analysis of notch and TGF- $\beta$ signaling expression in different stages of disease progression during hepatitis B virus infection. Clin Transl Gastroenterol 2012;3:e23.

29 Martinon F, Mayor A, Tschopp J: The inflammasomes: guardians of the body. Annu Rev Immunol 2009;27:229-265.

30 Schroder K, Tschopp J: The inflammasomes. Cell 2010;140:821-832.

-31 Rathinam VA, Vanaja SK, Fitzgerald KA: Regulation of inflammasome signaling. Nat Immunol 2012;13:333342.

-32 Davis BK, Wen H, Ting JP: The inflammasome NLRs in immunity, inflammation, and associated diseases. Annu Rev Immunol 2011;29:707-735.

33 Boaru SG, Borkham-Kamphorst E, Tihaa L, Haas U, Weiskirchen R: Expression analysis of inflammasomes in experimental models of inflammatory and fibrotic liver disease. J Inflamm (Lond) 2012;9:49.

34 Szabo G, Csak T: Inflammasomes in liver diseases. J Hepatol 2012;57:642-654.

-35 Csak T, Ganz M, Pespisa J, Kodys K, Dolganiuc A, Szabo G: Fatty acid and endotoxin activate inflammasomes in mouse hepatocytes that release danger signals to stimulate immune cells. Hepatology 2011;54:133144.

-36 Watanabe A, Sohail MA, Gomes DA, Hashmi A, Nagata J, Sutterwala FS, et al: Inflammasome-mediated regulation of hepatic stellate cells. Am J Physiol Gastrointest Liver Physiol 2009;296:G1248-G1257.

-37 Imaeda AB, Watanabe A, Sohail MA, Mahmood S, Mohamadnejad M, Sutterwala FS, et al: Acetaminophen-induced hepatotoxicity in mice is dependent on Tlr9 and the Nalp3 inflammasome. J Clin Invest 2009;119:305-314.

-38 Miura K, Kodama Y, Inokuchi S, Schnabl B, Aoyama T, Ohnishi H, et al: Toll-like receptor 9 promotes steatohepatitis by induction of interleukin-1beta in mice. Gastroenterology 2010;139:323-334.

-39 Ganz M, Csak T, Nath B, Szabo G: LPS stimulation induces and activates the Nalp3 inflammasome in the liver. World J Gastroenterol 2011;17:4772-4778.

40 Burdette D, Haskett A, Presser L, McRae S, Iqbal J, Waris G: Hepatitis C virus activates interleukin-1 $\beta$ via caspase-1-inflammasome complex. J Gen Virol 2012;93:235-246.

41 Petrasek J, Bala S, Csak T, Lippai D, Kodys K, Menashy V, et al: IL-1 receptor antagonist ameliorates inflammasome-dependent alcoholic steatohepatitis in mice. J Clin Invest 2012;122:3476-3489.

42 Henao-Mejia J, Elinav E, Jin C, Hao L, Mehal WZ, Strowig T, et al: Inflammasome-mediated dysbiosis regulates progression of NAFLD and obesity. Nature 2012;482:179-185.

43 Svegliati-Baroni G, Faraci G, Fabris L, Saccomanno S, Cadamuro M, Pierantonelli I, et al: Insulin resistance and necroinflammation drives ductular reaction and epithelial-mesenchymal transition in chronic hepatitis C. Gut 2011;60:108-115.

-44 Falkowski O, An HJ, Ianus IA, Chiriboga L, Yee H, West AB, et al: Regeneration of hepatocyte 'buds' in cirrhosis from intrabiliary stem cells. J Hepatol 2003;39:357-364.

45 Fausto N, Campbell JS, Riehle KJ: Liver regeneration. J Hepatol 2012;57:692-694.

-46 Gouw AS, Clouston AD, Theise ND: Ductular reactions in human liver: diversity at the interface. Hepatology 2011;54:1853-1863.

47 Zhou H, Rogler LE, Teperman L, Morgan G, Rogler CE: Identification of hepatocytic and bile ductular cell lineages and candidate stem cells in bipolar ductular reactions in cirrhotic human liver. Hepatology 2007;45:716-724.

48 Hanna RF, Aguirre DA, Kased N, Emery SC, Peterson MR, Sirlin CB: Cirrhosis-associated hepatocellular nodules: correlation of histopathologic and MR imaging features. Radiographics 2008;28:747-769.

49 Lin WR, Lim SN, McDonald SA, Graham T, Wright VL, Peplow CL, et al: The histogenesis of regenerative nodules in human liver cirrhosis. Hepatology 2010;51:1017-1026.

50 Wright TL: Regenerating nodules - are they premalignant lesions? Hepatology 1991;13:1254-1255.

51 Ng CH, Chan SW, Lee WK, Lai L, Lok KH, Li KK, Luk SH, Szeto ML: Hepatocarcinogenesis of regenerative and dysplastic nodules in Chinese patients. Hong Kong Med J 2011;17:11-19.

52 Hernandez-Gea V, Toffanin S, Friedman SL, Llovet JM: Role of the microenvironment in the pathogenesis and treatment of hepatocellular carcinoma. Gastroenterology 2013;144:512-527.

53 Seitz HK, Stickel F: Risk factors and mechanisms of hepatocarcinogenesis with special emphasis on alcohol and oxidative stress. Biol Chem 2006;387:349-360.

54 Missiha SB, Ostrowski M, Heathcote EJ: Disease progression in chronic hepatitis C: modifiable and nonmodifiable factors. Gastroenterology 2008;134:1699-1714.

-55 Bataller R, North KE, Brenner DA: Genetic polymorphisms and the progression of liver fibrosis: a critical appraisal. Hepatology 2003;37:493-503.

-56 Powell EE, Edwards-Smith CJ, Hay JL, Clouston AD, Crawford DH, Shorthouse C, et al: Host genetic factors influence disease progression in chronic hepatitis. Hepatology 2000;31:828-833. 
57 Huang H, Shiffman ML, Friedman S, Venkatesh R, Bzowej N, Abar OT, et al: A 7 gene signature identifies the risk of developing cirrhosis in patients with chronic hepatitis C. Hepatology 2007;46:297-306.

58 Marcolongo M, Young B, Dal Pero F, Fattovich G, Peraro L, Guido M, et al: A seven-gene signature (cirrhosis risk score) predicts liver fibrosis progression in patients with initially mild chronic hepatitis C. Hepatology 2009;50:1038-1044.

59 Minouchi K, Kaneko S, Kobayashi K: Mutation of p53 gene in regenerative nodules in cirrhotic liver. J Hepatol 2002;37:231-239.

60 Calado RT, Brudno J, Mehta P, Kovacs JJ, Wu C, Zago MA, et al: Constitutional telomerase mutations are genetic risk factors for cirrhosis. Hepatology 2011;53:1600-1607.

61 Hartmann D, Srivastava U, Thaler M, Kleinhans KN, N'kontchou G, Scheffold A, et al: Telomerase gene mutations are associated with cirrhosis formation. Hepatology 2011;53:1608-1617.

62 Di Gioia S, Bianchi P, Destro A, Grizzi F, Malesci A, Laghi L, et al: Quantitative evaluation of RASSF1A methylation in the non-lesional, regenerative and neoplastic liver. BMC Cancer 2006;6:89.

$63 \mathrm{Um} \mathrm{TH}, \mathrm{Kim}$ H, Oh BK, Kim MS, Kim KS, Jung G, et al: Aberrant CpG island hypermethylation in dysplastic nodules and early HCC of hepatitis B virus-related human multistep hepatocarcinogenesis. J Hepatol 2011;54:939-947.

64 Guichard C, Amaddeo G, Imbeaud S, Ladeiro Y, Pelletier L, Maad IB, et al: Integrated analysis of somatic mutations and focal copy-number changes identifies key genes and pathways in hepatocellular carcinoma. Nat Genet 2012;44:694-698.

65 Huang J, Deng Q, Wang Q, Li KY, Dai JH, Li N, et al: Exome sequencing of hepatitis B virus-associated hepatocellular carcinoma. Nat Genet 2012;44:1117-1121.

66 Bose S, Sakhuja P, Bezawada L, Agarwal AK, Kazim SN, Khan LA, et al: Hepatocellular carcinoma with persistent hepatitis B virus infection shows unusual downregulation of Ras expression and differential response to Ras mediated signaling. J Gastroenterol Hepatol 2011;26:135-144.

-67 Shay JW, Wright WE: Hayflick, his limit, and cellular ageing. Nat Rev Mol Cell Biol 2000;1:72-76.

-68 Yaswen P, Campisi J: Oncogene-induced senescence pathways weave an intricate tapestry. Cell 2007;128:233-234.

69 Adams PD: Healing and hurting: molecular mechanisms, functions, and pathologies of cellular senescence. Mol Cell 2009;36:2-14

-70 Krizhanovsky V, Yon M, Dickins RA, Hearn S, Simon J, Miething C, et al: Senescence of activated stellate cells limits liver fibrosis. Cell 2008;134:657-667.

71 Schnabl B, Purbeck CA, Choi YH, Hagedorn CH, Brenner D, et al: Replicative senescence of activated human hepatic stellate cells is accompanied by a pronounced inflammatory but less fibrogenic phenotype. Hepatology 2003;37:653-664.

72 Kang TW, Yevsa T, Woller N, Hoenicke L, Wuestefeld T, Dauch D, et al: Senescence surveillance of pre-malignant hepatocytes limits liver cancer development. Nature 2011;479:547-551.

73 Wiemann SU, Satyanarayana A, Tsahuridu M, Tillmann HL, Zender L, Klempnauer J, et al: Hepatocyte telomere shortening and senescence are general markers of human liver cirrhosis. FASEB J 2002;16:935-942.

-74 Wang Z, Lin H, Hua F, Hu ZW: Repairing DNA damage by XRCC6/KU70 reverses TLR4-deficiency-worsened HCC development via restoring senescence and autophagic flux. Autophagy 2013;9:925-927.

$>75$ Iredale JP: Models of liver fibrosis: exploring the dynamic nature of inflammation and repair in a solid organ. J Clin Invest 2007;117:539-548.

76 Timchenko NA: Aging and liver regeneration. Trends Endocrinol Metab 2009;20:171-176.

-77 Ramakrishna G, Anwar T, Angara RK, Chatterjee N, Kiran S, Singh S: Role of cellular senescence in hepatic wound healing and carcinogenesis. Eur J Cell Biol 2012;91:739-747. 